\title{
13 Plasticity across Generations
}

\author{
Russell Bonduriansky \\ University of New South Wales
}

\section{CONTENTS}

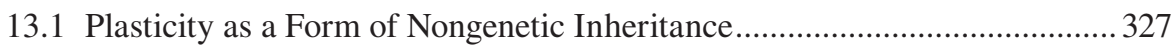

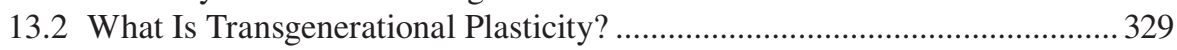

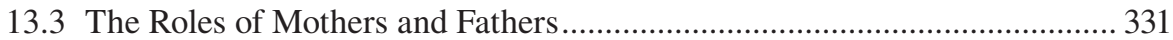

13.4 The Stability of Transgenerational Plasticity ................................................ 332

13.5 The Evolution of Adaptive Transgenerational Plasticity .................................334

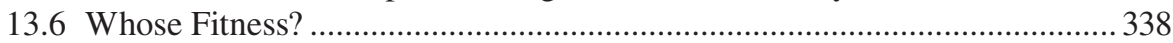

13.7 Why Transgenerational Plasticity Is Often Non-Adaptive ............................ 339

13.8 Can Transgenerational Plasticity Influence the Course of Adaptive

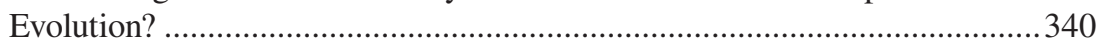

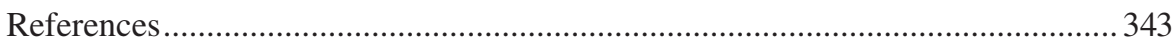

\subsection{PLASTICITY AS A FORM OF NONGENETIC INHERITANCE}

Previous chapters have outlined the classic concept of plasticity, its mechanistic basis, and its adaptive importance. Plasticity is the ability of an individual organism or genotype to respond in a consistent way to environmental conditions, either during development ('developmental plasticity') or during adulthood (sometimes called 'phenotypic flexibility,' Piersma and Drent 2003). All organisms are plastic in at least some of their traits, and some plastic responses represent evolved strategies that enhance fitness under certain conditions, while other plastic responses represent maladaptive effects of stress or pathology. Plasticity can operate through a variety of cellular, physiological, and behavioral mechanisms. Among these, epigenetic processes such as the modification of DNA methylation patterns and/or chromatin structure in certain genomic regions are believed to play a particularly important role (Duncan et al. 2014; Paksa and Rajagopal 2017). Such environment-induced epigenetic changes result in modified patterns of gene expression and thus mediate changes in phenotypic traits and fitness.

Plasticity in the classic sense involves individuals responding to environments that they experience during their lifetime ('within-generation plasticity'). For example, many organisms respond to predator cues by expressing defensive traits: plants grazed by herbivores can elevate investment in morphological and chemical 
defenses against herbivory (War et al. 2012), while Daphnia hatchlings that encounter chemical cues associated with predation express defensive spines (Laforsch and Tollrian 2004). However, such plastic effects were long assumed to be lost between generations because it was believed that genes are the only factors that are transmitted to offspring and that can influence offspring development. Since genes could not be altered in consistent ways by the environment, it followed that environmental effects on bodily traits (the phenotype) could not be transmitted across generations, and 'Lamarckian' processes such as the 'inheritance of acquired traits' were not possible (for discussion of the history of these ideas see Bonduriansky 2012; Bonduriansky and Day 2018). According to this classic view, you can damage your own health by avoiding exercise, eating an unhealthy diet, or smoking, but your children and grandchildren will not be born any less healthy as a result. Rather, each individual's traits are shaped by its own unique genotype (the combination of genetic alleles determined by the random lottery of recombination) and by the environment that it experiences during its own development and lifetime. Environments experienced by ancestors matter only in so far as, by imposing natural selection on genetic variation, they have altered the frequencies of alleles in subsequent generations.

Classic theory allowed only two exceptions to this view. First, in some organisms, the mother's phenotype clearly forms an important part of the developmental environment in which the genomes of her offspring are expressed. Maternal traits involved in offspring care and provisioning were therefore assumed to shape some aspects of development, resulting in maternal effects (Bernardo 1996; Mousseau and Fox 1998). Second, in humans (and perhaps a few other cognitively complex, social animals, such as apes, monkeys, and dolphins), aspects of behavior were assumed to be shaped by culture, which could be transmitted across generations independently of genetic alleles (Mesoudi 2011).

Yet, it is now clear that many of the epigenetic and physiological mechanisms involved in plastic responses can also affect gamete formation and offspring development. Many nongenetic factors are transmitted across generations alongside genes, and variation in such nongenetic factors can have important effects on offspring phenotypes and fitness. Because many aspects of reproductive physiology are highly sensitive to environmental influence and can respond in consistent ways to particular environmental factors or stresses, some effects of ancestors' environment (including, as it turns out, effects of physical activity, diet, and smoking) can be transmitted to subsequent generations. Such effects are called transgenerational plasticity (Galloway and Etterson 2007; Bell and Hellmann 2019).

Transgenerational plasticity is a form of nongenetic inheritance that includes classic maternal effects such as effects of maternal diet (Bernardo 1996; Mousseau and Fox 1998) and can encompass effects of learning and culture in some mammals, birds, and other animals in which individuals can adjust their behavior to the environmental conditions that they encounter and then pass on those behaviors to their offspring via learning (Jesmer et al. 2018; Aplin 2019). However, the current understanding of transgenerational plasticity is much broader: transgenerational plasticity can encompass a much wider range of environmental factors and phenotypic effects than hitherto appreciated; can involve effects of environments experienced by both mothers and fathers; and, in some cases, can involve effects of environments 
experienced by grandparents and even more remote ancestors. Transgenerational plasticity is therefore a phenomenon that was not recognized in classic Modern Synthesis theory, and its scope and importance have only recently come to be widely recognized by evolutionary ecologists.

Transgenerational plasticity is a young research field: many questions remain to be answered, and there is a great deal of controversy surrounding the fitness effects and evolutionary implications of this phenomenon (for a discussion of the history and current state of this controversy, see Bonduriansky and Day 2018). In this chapter, I will attempt to clarify some aspects of this controversy by considering the nature of transgenerational plasticity and its potential to influence the course of evolution. In particular, I will consider whether it is reasonable to assume that transgenerational plasticity is typically adaptive and whether transgenerational plasticity has the potential to provide variation that natural selection can act on to drive adaptive evolution. I will argue that, like classic plasticity, transgenerational plasticity need not be adaptive, and researchers should be very careful in inferring that an observed pattern of transgenerational plasticity represents an evolved, adaptive response. Yet, I will also argue that non-adaptive instances of transgenerational plasticity can be just as interesting and as important as adaptive transgenerational plasticity, and have the potential to play a substantial role in adaptive evolution.

\subsection{WHAT IS TRANSGENERATIONAL PLASTICITY?}

Nongenetic inheritance (or parental effects in the broadest sense) involves effects of ancestors on descendants that are not mediated by the transmission of genetic alleles. Transgenerational plasticity is the sub-set of nongenetic inheritance that involves nongenetic transmission of effects consistently induced by particular environmental factors (Bell and Hellmann 2019). Transgenerational plasticity excludes effects that genes expressed in parents have on offspring (indirect genetic effects), as well as effects of nongenetic factors that are unaffected by environment or affected in unpredictable ('random') ways (analogous to effects of mutagens on DNA sequences).

Many examples of transgenerational plasticity have been reported, affecting health, behavior, morphology, life history, and fitness (for examples see Bonduriansky and Day 2018; Bell and Hellmann 2019). For example, in some species, exposure to predator cues can induce the expression of defensive traits not only in individuals that are directly exposed to those cues but also in their offspring. Thus, plants attacked by herbivores can produce offspring with elevated defenses (Colicchio 2017), and some Daphnia exposed to predator cues produce offspring that express defensive spines even in the absence of predators (Agrawal et al. 1999). In such cases, the effects on offspring or grand-offspring are very similar to direct effects of environment on individual development. Such cases are clearly analogous to plasticity in the classic sense and may be mediated by the same proximate factors. For example, predator cues might induce changes in DNA methylation or chromatin structure that cause the expression of defensive traits, and those same epigenetic changes may occur in the germ-line and induce similar developmental effects (albeit not necessarily of the same magnitude) in offspring. However, in other cases, the direct effect of a given environmental factor may be quite different from its effect on offspring. 
For example, many fish, including sticklebacks, respond to predator cues by adopting risk-minimizing behaviors (Wund et al. 2015), but the offspring of predator-exposed sticklebacks do the opposite, behaving in ways that increase rather than reduce their risk of being eaten (McGhee et al. 2012). Such counterintuitive effects appear to be non-adaptive and could result from the transmission of stress-induced changes in epigenetic factors such as DNA methylation. Many other environmental factors, such as toxins, diet, and social environment, can also affect offspring development (Bonduriansky and Day 2018; Bell and Hellmann 2019).

While studies at the whole-organism level have revealed many examples of transgenerational plasticity involving a great variety of environmental factors and organismal traits, the proximate mechanisms mediating such effects are still poorly understood in most systems. Transgenerational plasticity involves a complex causal chain linking an environmental exposure to changes in parental reproductive physiology, a resulting change in some factor that is transmitted from parent to offspring (such as a DNA methylation pattern, small RNA, histone, hormone, or nutrient), and a consequent cascade of developmental effects that induce a change in offspring phenotype (Figure 13.1). This process is often viewed as a signaling system whereby parents transmit 'information' or 'cues' to their offspring so as to enhance offspring

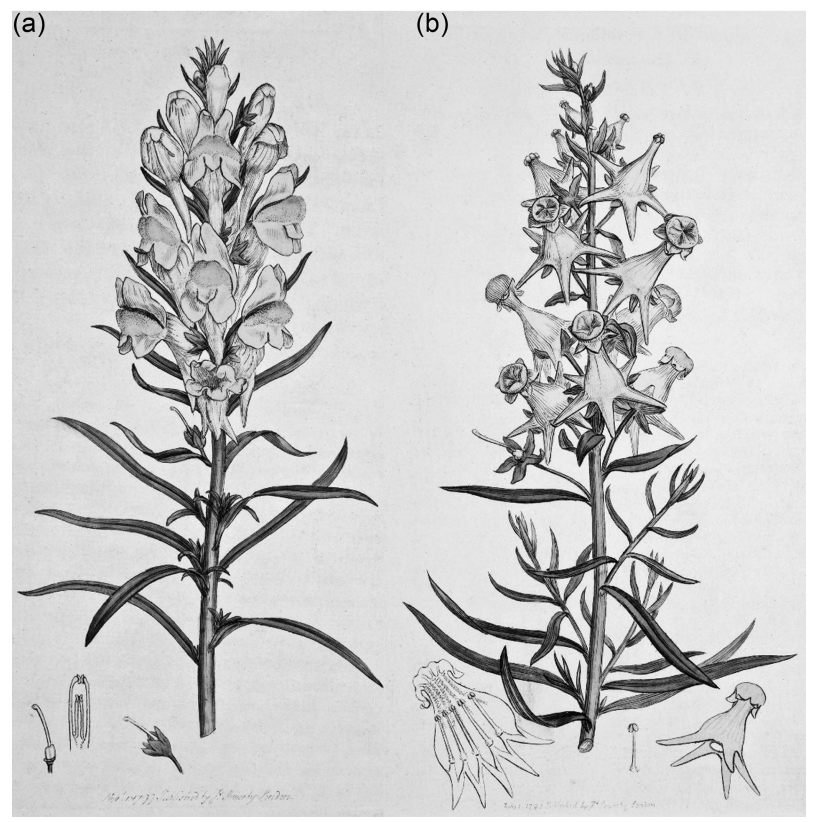

FIGURE 13.1 The normal (a) and peloric (b) flower forms of toadflax (Linaria vulgaris), first described by Carl Linnaeus. Peloric flower morphology is caused by reduced DNA methylation of the $L c y c$ gene-an epigenetic variant ('epiallele') that can be transmitted from parent to offspring over several generations. It is not known whether this epigenetic factor can be induced by the environment, and thus whether this famous example of nongenetic inheritance represents an instance of transgenerational plasticity. (Illustrations by James Sowerby, courtesy of the John Innes Foundation.) 
fitness. Yet, many instances of transgenerational plasticity appear to involve nonadaptive or even deleterious effects, and offspring can be selected to disregard such parental effects (Bernardo 1996; Uller 2008; Bell and Hellmann 2019). To my knowledge, this causal chain has not yet been fully characterized in any organism, but studies on model organisms such as plants, mice, nematode worms, and other organisms are providing an increasingly detailed picture of parts of this chain. For example, a recent study on mice shows that psychological stress can affect the RNA content of membrane-enclosed 'extracellular vesicles' that are released by cells throughout the body and within the epididymis (where sperm undergo maturation), that these vesicles can transport their RNA cargo to developing spermatozoa, and that ovules fertilized by such spermatozoa develop into offspring with altered growth and stress responses (Chan et al. 2020). This study provides an unusually detailed picture of how stressful experiences can affect the RNA cargo of sperm. Yet, many steps in the causal chain remain to be filled in, including how psychological stress induces consistent changes in extracellular vesicle content, and how the transmission of these factors affects embryonic development so as to produce the observed phenotypic effects. Interestingly, a recent study shows that RNA can be transported from the mouse brain to the testes and then transmitted to embryos, suggesting a potential means for stress- or experience-induced changes in gene expression in the brain to lead to altered RNA content in the germ-line (O'Brien et al. 2020).

\subsection{THE ROLES OF MOTHERS AND FATHERS}

Maternal transgenerational plasticity (i.e., environment-induced 'maternal effects') has been recognized for a long time, although its scope and importance in ecology, evolution, and health was under-appreciated until quite recently (Kirkpatrick and Lande 1989; Bernardo 1996). Maternal effects were long treated as a nuisance factor in quantitative-genetic studies, but have now come to be seen as a fascinating and important phenomenon in their own right. Given the intimate physiological and behavioral interaction between offspring and their mothers in many animals, many examples of maternal effects are mediated by variation in maternal care. For example, in rats, variation in maternal behavior (e.g., induced by maternal stress) can induce epigenetic (DNA methylation) changes in the offspring brain, and these changes can persist throughout life and affect the adult behavior and stress responses of the offspring (Weaver et al. 2004; Champagne and Meaney 2007).

In contrast to maternal effects, paternal transgenerational plasticity was almost entirely ignored and, indeed, assumed to be virtually impossible in most systems until fairly recently. Because males do not interact with their offspring in most species (including classic model animals such as Drosophila and mice), it was assumed that males could not influence their offspring nongenetically. Yet, a variety of such mechanisms are now recognized (including modification of methylation and chromatin structure in sperm-borne DNA, noncoding RNA in sperm and seminal fluid, seminal proteins, and other factors), and many examples of paternal transgenerational plasticity have now come to light (Crean and Bonduriansky 2014; Soubry et al. 2014). For example, as mentioned above, male mice that experience severe stress produce offspring with altered growth and stress responses, and these effects 
are mediated by molecules transmitted in the sperm (Chan et al. 2020). Paternal transgenerational plasticity may yet turn out to be as widespread and important as maternal transgenerational plasticity. Although opportunities for father-offspring influence are limited in most animals because males do not provision, nurture, or interact with their offspring (Crean and Bonduriansky 2014), the sperm epigenome may be highly susceptible to environment-induced modification and damage, potentially enabling a wide variety of epigenetically mediated effects of paternal environment to be transmitted through the male germ-line (Pembrey et al. 2014).

It is now well established that both maternal and paternal health can have profound effects on the development and health of the offspring. For example, in rats, offspring metabolism is strongly affected by paternal diet (Anderson et al. 2006; $\mathrm{Ng}$ et al. 2010). Paternal obesity can also affect children's health in humans via paternal influence on children's lifestyle as well as sperm-borne epigenetic factors (Donkin et al. 2016; Sharp and Lawlor 2019). Likewise, in both rodents and humans, there is abundant evidence that offspring metabolism can be strongly affected by maternal obesity (Amarger et al. 2014; Dominguez-Salas et al. 2014; Kamimae-Lanning et al. 2014). Indeed, in humans and other mammals, there is potential for complex interactions and reinforcement between effects of parental environment transmitted through the germ-line, intra-uterine effects mediated by maternal health, and postnatal effects mediated by maternal (and, in some species, also paternal) behavior (Archer 2015).

\subsection{THE STABILITY OF TRANSGENERATIONAL PLASTICITY}

Maternal effects were long assumed to be mediated by direct mother-offspring interaction in utero and via maternal care. However, in mammals, the germ-line develops early in embryonic development, and female embryos in the womb already contain their own eggs. These eggs could be subject to the same intra-uterine effects as the embryonic soma, resulting in grand-maternal effects (Youngson and Whitelaw 2008). While intra-uterine effects are limited to two generations, some epigenetic processes have the potential to mediate transgenerational plasticity across multiple generations (Jablonka and Raz 2009; Boskovic and Rando 2018). For example, some variable patterns of DNA methylation ('epialleles') — such as the peloric epiallele of the Lcyc gene that causes altered flower form in toadflax (Linaria vulgaris) (Figure 13.1) - appear to be stable over several generations (Cubas et al. 1999). Other nongenetic factors, such as self-regenerating gene expression loops, have the potential to persist over multiple generations as well (Jablonka and Raz 2009). Multigenerational transgenerational plasticity can occur through patrilines as well as matrilines. For example, in mice and humans, exposure to toxins can be transmitted through patrilines to grand-offspring and beyond (Chen et al. 2006; Pembrey et al. 2014; Skinner 2014). Likewise, in sticklebacks, exposure to predators induces grand-paternal effects that also differ by offspring sex (Hellmann et al. 2020). Such multigenerational examples of transgenerational plasticity could have very interesting implications for phenotypic variation and adaptive evolution (discussed below), but relatively few experimental studies have investigated the potential for transgenerational plasticity to persist over more than two generations, or for environmental effects that 
act over multiple generations to accumulate and interact (Rutkowska et al. 2020). The proximate mechanisms that mediate the transmission of environmental effects across multiple generations remain poorly understood, although considerable progress in the understanding of such mechanisms has come from work on nematode worms (Greer et al. 2010; Greer et al. 2011; Klosin et al. 2017; Rechavi and Lev 2017). Many patterns of multigenerational transgenerational plasticity have been observed in empirical studies, and a wide range of proximate mechanisms might be involved in generating these patterns (Bell and Hellmann 2019).

The potential for effects of environment to be transmitted over multiple generations is of interest because such effects could provide heritable variation on which natural selection can act (Day and Bonduriansky 2011; Klironomos et al. 2013; Furrow 2014). However, even effects that fade out after a single generation could still be very important in many evolutionary contexts. For example, such effects could play an important role in allowing populations to persist in fluctuating environments (Lachmann and Jablonka 1996; Dey et al. 2016; see also Diamond and Martin 2021 in this volume). Transient effects of parental environment also have the potential to play important roles in coevolution between the sexes (Bonduriansky and Day 2013) and between hosts and parasites (Qutob et al. 2013; Gijzen et al. 2014; Bonduriansky and Day 2018), gene-culture coevolution (Feldman and Cavalli-Sforza 1989; Richerson and Boyd 2005), and even in speciation and diversification (Jablonka and Raz 2009; Pfennig and Servedio 2012). Of course, as noted above, the effects of parental environment on offspring can also have very important implications for human health. In Box 13.1, I discuss some of the terminology used to describe transgenerational plasticity.

\section{BOX 13.1 DESCRIBING TRANSGENERATIONAL PLASTICITY}

Because transgenerational plasticity has only recently become a major focus of research, terminology relating to this phenomenon is still evolving and the literature is rife with confusing jargon. The effects of parents on their offspring are sometimes called 'intergenerational' while effects spanning more than one generation are called 'transgenerational.' While this terminology is still widely used in studies on transgenerational plasticity (especially in mammals), its utility is limited. Examples of transgenerational plasticity effects spanning three, four, five, or more generations can be found in the literature but, given that most studies of transgenerational plasticity span a single generation, the terms used to describe the observed effects are often based on the number of generations over which a given effect has been investigated rather than the number of generations over which the effect can actually be transmitted. Moreover, the potential stability of a given effect over multiple generations could depend on the environment in which descendants are assayed, or even the genotype of the organisms used. To avoid confusion, researchers could simply report the number of generations over which a given effect has been studied or, in a multi-generation study in which an effect was observed to fade 
out after a certain number of generations, report the number of generations over which the effect could be detected.

Likewise, factors such as DNA methylation could mediate transgenerational plasticity in diverse ways. In particular, studies on transgenerational plasticity should clearly differentiate the transmitted factor-that is, the environment-dependent entity (e.g., DNA methylation pattern, small RNA, hormone, nutrient, symbiont, or learned behavior) that is passed from parent to offspring through the germ-line, the intrauterine environment or posthatching/post-partum parental care-from the consequent developmental cascade in the offspring (such as changes in offspring growth rate, or altered patterns of DNA methylation in the offspring brain) that brings about changes in offspring phenotype. For example, an effect mediated by the transmission of an environment-dependent pattern of DNA methylation through the germline can be regarded as an instance of epigenetic transmission, while an effect mediated by a parental behavior that induces changes in DNA methylation in the offspring brain can be regarded as an instance of behavioral transmission. The nature of the transmitted factor and the developmental cascade can have important implications for the environmental inducibility, stability across generations, sex-specificity, and taxonomic distribution of transgenerational effects (Bonduriansky and Day 2018).

\subsection{THE EVOLUTION OF ADAPTIVE TRANSGENERATIONAL PLASTICITY}

Like within-generation plasticity (see Pfennig 2021 in this volume), transgenerational plasticity can evolve as a facultative, fitness-enhancing strategy. The most widely recognized form of adaptive transgenerational plasticity is the ability of mothers to adjust the development of their offspring and thereby enhance offspring fitness in a similar environment. For example, mothers that experience predator cues can benefit by producing offspring that are primed to express anti-predator defenses (Agrawal et al. 1999), and solitary ascidians (Styela plicata) that develop at low density sire offspring that have enhanced performance under low-density conditions (Crean et al. 2013). Recognized at least since the 1990s (Bernardo 1996), such effects are often called 'anticipatory' parental effects (Marshall and Uller 2007). Anticipatory effects will have a net positive effect on the fitness of both mothers and their offspring when the environment experienced by parents is generally predictive of the environment experienced by offspring. According to theory, anticipatory transgenerational plasticity should therefore evolve when the environment fluctuates predictably between alternative states (e.g., predators abundant/predators rare, hot/ cold, wet/dry), and the period of fluctuations is sufficiently long to generate a temporal autocorrelation across generations (Lachmann and Jablonka 1996; Proulx and Teotonio 2017). These predictions were supported by an experimental study showing that anticipatory transgenerational plasticity evolves rapidly in the nematode worm 
Caenorhabditis elegans when the environment fluctuates predictably between two states (high/low oxygen), but does not evolve when environmental fluctuations are random (Dey et al. 2016). While the evolution of anticipatory transgenerational plasticity is usually considered in the context of temporal fluctuations between environmental states, the same logic applies in the case of spatial heterogeneity. For example, if individuals can exploit different host types, and parents can predict the host type on which their offspring will develop, then selection might favor anticipatory effects that enhance offspring performance on the expected host type (Fox et al. 1997). Anticipatory transgenerational plasticity is usually detected using experiments that manipulate both parental and offspring environments. The prediction is that offspring performance is enhanced when the offspring develop in the same environment as their parents (Figure 13.2a; but see Engqvist and Reinhold 2016).

Anticipatory transgenerational plasticity represents an alternative to withingeneration plasticity: individuals can enhance their fitness either by responding to environmental conditions that they themselves experience, or to environmental conditions experienced by their parents. But which option is better? The broad insight from theoretical studies is that within-generation plasticity can be advantageous because it allows for a more immediate response to prevailing conditions, while anticipatory transgenerational plasticity can be favored because it can give offspring a developmental head start (Uller 2008). For example, if developmental response to predator cues takes time, offspring that encounter such cues may not be able to develop defenses quickly enough to avoid predation. Transgenerational plasticity may allow an anti-predator defense to begin developing earlier, even before offspring themselves actually encounter predator cues, or develop the sensory organs required to detect such cues. On the other hand, environmental conditions that prevailed during the previous generation can be an unreliable cue to the conditions that offspring will experience, and fitness is reduced if offspring end up developing in a mismatched environment (for example, investing in costly defenses when predators are absent). The less predictable the environment is across generations, the greater the advantage of within-generation plasticity over anticipatory transgenerational plasticity. Depending on the balance of these costs and benefits, selection could therefore favor within-generation plasticity only, transgenerational plasticity only, some combination of within-generation plasticity and transgenerational plasticity, or no plasticity at all (Leimar and McNamara 2015; McNamara et al. 2016).

The physiological, sensory, and neural mechanisms involved in detecting and assessing environmental cues are expected to impose costs, although the nature and magnitude of such costs remain poorly known (Van Buskirk and Steiner 2009; Murren et al. 2015; see also Snell-Rood and Ehlman 2021 in this volume). As noted above, plastic responses can also be prone to error when environmental cues are unreliable. The epigenetic machinery that mediates transgenerational plasticity is likely to impose metabolic costs as well, although the nature and magnitude of such costs are poorly understood (Macartney et al. 2018). If transgenerational plasticity is indeed costly, and especially if environmental conditions remain relatively constant for many generations, reducing the benefits of transgenerational plasticity, then selection could favor the replacement of transgenerational plasticity by an 
(a)

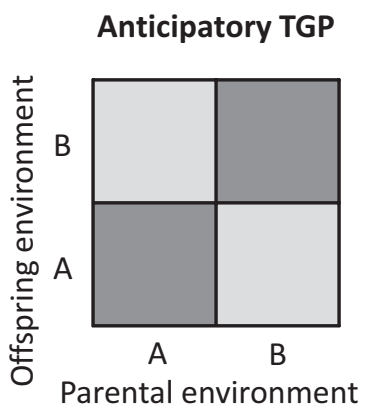

(b)

Condition-transfer TGP

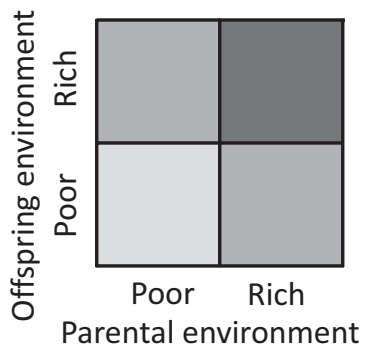

(c) Complex offspring $x$ parental environment interaction

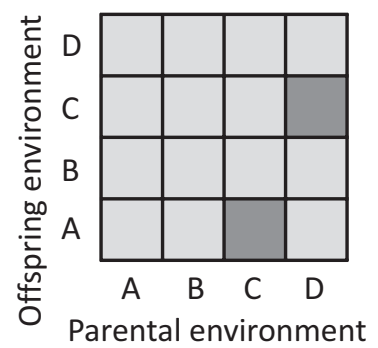

FIGURE 13.2 Potential effects of parental environment and offspring environment on offspring fitness (darker gray = higher fitness): (a) anticipatory transgenerational plasticity (TGP) is reflected in an interaction between parental and offspring environment, whereby offspring perform well when they develop in a similar environment to that experienced by their parents, but poorly when offspring environment is mismatched to the parental environment; (b) condition-transfer transgenerational plasticity is reflected in enhanced performance of offspring of high-condition parents across a broad range of environments, and perhaps especially in resource-poor environments (where, by contrast, an anticipatory effect would be expected to result in reduced performance of offspring produced by high-condition parents); (c) a complex interaction of parental and offspring environments where certain environment combinations appear to be associated with enhanced offspring performance. Such complex interactions are unlikely to represent evolved, adaptive transgenerational plasticity unless the particular environment combinations that are associated with enhanced offspring performance are commonly encountered in nature. 
environment-independent developmental program — an evolutionary process called 'genetic assimilation' (see Scheiner and Levis 2021 in this volume). If a formerly fluctuating environment becomes stable, the most advantageous phenotype that formerly resulted from an epigenetic factor can therefore become genetically 'fixed' (Pál and Miklós 1999). For example, if predator abundance formerly fluctuated between high and low values but eventually stabilized at a high value, selection might favor all individuals developing anti-predator defenses without having to respond to either parental cues or the current environment.

While anticipatory effects are the most widely recognized form of adaptive transgenerational plasticity, another type of adaptive transgenerational plasticity that might be equally widespread and important is the transmission of conditiondependent nongenetic factors from parents to their offspring ('condition transfer'; Qvarnström and Price 2001; Bonduriansky and Crean 2018). Condition transfer can occur when gamete or seminal fluid production, parental investment, or other parental traits that affect offspring are condition-dependent in their expression, resulting in a correlation between parental and offspring condition. For example, Atlantic salmon (Salmo salar) that spend longer at sea and thereby grow to a larger size prior to spawning produce larger eggs and longer and heavier offspring (Van Leeuwen et al. 2016). Similarly, in the marine fish Acanthochromis polyacanthus, high-condition parents produced offspring that were longer and heavier and had enhanced viability (Donelson et al. 2009). Some degree of condition transfer is probably inevitable because reproduction is costly, and low-condition parents may be unable to provide as well for their offspring as high-condition parents can. Condition transfer is obviously disadvantageous when parents are in poor condition, and some authors have therefore argued that condition transfer should be regarded as nonadaptive. However, condition transfer is likely to enhance fitness on average. Indeed, natural selection may often drive the evolution of heightened condition-dependence of reproductive traits, for the same reasons that selection often favors heightened condition-dependence of other costly fitness-related traits, such as sexual signals. High-condition individuals leave more progeny on average than do low condition individuals, and any strategy that benefits high-condition individuals will therefore be under positive net selection. High-condition parents will benefit by transferring condition-enhancing nongenetic factors to their offspring because this will tend to enhance offspring performance across a broad range of environments, and selection is therefore expected to promote heightened condition-dependence in many reproductive traits (Bonduriansky and Crean 2018).

Detecting condition transfer involves showing that offspring of high-condition parents perform better across a broad range of environments than do offspring of low-condition parents. Like anticipatory transgenerational plasticity, condition transfer can be detected using experiments where the quality of parental and offspring environments is manipulated in a fully crossed design. However, unlike anticipatory transgenerational plasticity, condition-transfer transgenerational plasticity predicts that high-condition parents (i.e., parents reared or maintained in a resource-rich environment) will produce offspring that perform better across a range of environments, and do not suffer fitness costs when offspring environment is mismatched to parental environment. In particular, while anticipatory transgenerational plasticity predicts 
that offspring of low-condition parents would outperform offspring of high-condition parents in a resource-poor environment, condition-transfer transgenerational plasticity predicts that offspring of high-condition parents would have an advantage in both resource-poor and resource-rich environments (Figure 13.2b; Donelson et al. 2009; but see Engqvist and Reinhold 2016).

\subsection{WHOSE FITNESS?}

Unless offspring are clones of their parents, the fitness interests of parents and their offspring are non-identical. Parents are selected to shape offspring development in a way that enhances parental fitness, and Marshall and Uller (2007) therefore defined adaptive parental effects as effects that enhance parental fitness, irrespective of effects on the fitness of individual offspring. For example, mothers in high condition might be selected to increase offspring number while reducing offspring body size, a strategy that might enhance maternal fitness while reducing the fitness of individual offspring. Such a pattern is observed in the mosquitofish (Gambusia holbrooki), where mothers reared at low density and abundant food produced more and smaller offspring than mothers reared at high density and limited food (O'Dea et al. 2015). Similarly, in wild chimpanzees, some mothers in high condition appear to wean offspring early in order to produce their next offspring sooner (Thompson et al. 2016). However, offspring are selected to pursue developmental strategies that maximize their own fitness, and selection should therefore favor offspring strategies that counteract parental effects (such as reduced investment per offspring) that reduce offspring fitness. In some cases, transgenerational plasticity could therefore be shaped by parent-offspring conflict (Uller 2008; Kuijper and Johnstone 2018). A full understanding of adaptive transgenerational plasticity requires taking the interests of both parents and their offspring into consideration.

Patterns of transgenerational plasticity could also be shaped by the differing interests of female and male offspring. If the sexes experience different patterns of selection, then transgenerational plasticity could be subject to sexual conflict. For example, if males and females typically experience different environmental conditions and these conditions affect the germ-line epigenome, offspring may receive conflicting epigenetic signals from their mothers and fathers, and may be selected to respond differently to these signals. If sex-specific ecology is relatively stable over generations then selection will generally favor offspring that respond to epigenetic signals received from the same-sex parent while ignoring epigenetic signals received from the opposite-sex parent. Genes that control offspring responses to epigenetic signals transmitted from their parents could therefore be subject to sexually antagonistic selection, and this could result in a range of adaptive or maladaptive patterns of transgenerational plasticity. Individual-based simulations show that, if a single locus controls offspring responses to parental signals, then adaptive transgenerational plasticity may fail to evolve because benefits to one sex will be balanced by costs to the other sex. However, if the locus is duplicated, or if sex-linked modifiers evolve, allowing offspring to respond only to epigenetic signals from their same-sex parent, then an adaptive, sexually dimorphic pattern of transgenerational plasticity may evolve (Burke et al. 2020). 


\subsection{WHY TRANSGENERATIONAL PLASTICITY IS OFTEN NON-ADAPTIVE}

It's tempting to assume adaptive function for all or most observed patterns of transgenerational plasticity, but this assumption is unwarranted and probably reflects a lingering bias in how researchers think about nongenetic versus genetic inheritance. Biologists are accustomed to the idea that much of the variation that is transmitted genetically is non-adaptive. Genetic mutations rarely enhance fitness. All gametes carry deleterious alleles, and zygotes incorporate such alleles into the offspring genome. Biologists rarely ask why deleterious alleles (as well as transposons and other 'junk DNA') are faithfully transmitted from parents to their offspring. We simply assume that all these DNA sequences are transmitted across generations because there is no way to filter out the good from the bad.

Most instances of nongenetic inheritance should be viewed in much the same way. Non-adaptive transgenerational plasticity occurs because many aspects of reproductive physiology are sensitive to environment and, as a result, many environment-dependent factors are transferred across generations as a byproduct of the reproductive process. As with genetic alleles, there may be no way to filter out deleterious factors from advantageous ones. There is ample evidence that old, sick, or malnourished parents tend to produce offspring in poor condition, and such effects are mediated by reduced quality of the gametes, seminal fluid, intra-uterine environment, or post-partum parental care. For example, environmental stressors such as toxins and endocrine disruptors (Skinner 2014), an unbalanced diet ( $\mathrm{Ng}$ et al. 2010; Kamimae-Lanning et al. 2014), or psychological stress (Mashoodh and Champagne 2014; Schmauss et al. 2014; Zannas et al. 2015) can cause epigenetic dysregulation. If some of these dysregulated epigenetic signatures occur in the germ-line, they could be transmitted to offspring. Moreover, if the ability to maintain the germ-line epigenome (i.e., the pattern of DNA methylation and chromatin structure across the genome) declines with age, environmental stressors may tend to have greater effects on the germ-line with advancing age (Monaghan and Metcalfe 2019). This could explain why older parents tend to produce offspring of lower condition (Wylde et al. 2019), and why the rate of decline in offspring condition with parental age can be modulated by the environment (Gribble et al. 2014). As noted above, non-adaptive or mal-adaptive transgenerational plasticity can also result from parent-offspring conflict or sexual conflict.

Many observed patterns of transgenerational plasticity are therefore probably non-adaptive or even mal-adaptive. Besides conflict between parents and offspring and between sexes (see previous section), non-adaptive patterns of transgenerational plasticity may often result from constraints on the evolution of reproductive physiology. Like within-generation plasticity, many instances of transgenerational plasticity reflect the transmission of pathology or stress and generally reduce the fitness of parents and their offspring. Recognizing the potential for non-adaptive transgenerational plasticity has implications for the interpretation of empirical results. If a seemingly adaptive effect is observed in a few treatment combinations, reflected in a complex parental $\times$ offspring environment interaction (Figure 13.2c), it's unlikely to have evolved by selection if that particular combination of ancestral and current 
environments rarely occurs. As a hypothetical example, a multifactorial experiment might show that, when parents are maintained in a hot environment and at high density, their offspring show enhanced performance when provided with protein-rich food. To determine whether this pattern can be plausibly interpreted as adaptive, it is necessary to study the ecology of natural populations and establish whether or not this particular combination of parental and offspring environments happens to be common in the wild, thereby providing ample opportunity for natural selection to act on this parental effect. If information on the correlation of parental and offspring environments in the wild is lacking, the pattern of transgenerational plasticity observed in such experiments should not be assumed to represent an adaptive effect. Of course, conclusions about the fitness effects of any form of transgenerational plasticity are subject to all the complexities and caveats involved in quantifying fitness, such as environment specificity, latent effects, and trade-offs (Bell and Hellmann 2019).

\subsection{CAN TRANSGENERATIONAL PLASTICITY INFLUENCE THE COURSE OF ADAPTIVE EVOLUTION?}

Nineteenth-century evolutionary theories, including the progressive concept of evolution proposed by Jean Baptiste Lamarck (1809) and the theory of 'descent with modification' via natural selection on heritable variation proposed by Charles Darwin $(1859,1871,1875)$, were based on the assumption that environment-induced variation could be transmitted to offspring. Darwin even suggested a detailed mechanism of heredity (called 'pangenesis') that enabled the transmission of environment-induced effects through the transfer of particles called 'gemmules' in eggs and sperm. However, with the advent of Mendelian genetics in the early 20th century, such 'inheritance of acquired traits' was deemed incompatible with the nature of genes and declared impossible. As I noted in Section 13.1, all forms of nongenetic inheritance-including transgenerational plasticity, as well as randomly mutating nongenetic factors, and indirect genetic effects-were therefore excluded from modern evolutionary theory. Although important evidence of nongenetic inheritance such as experimental evidence of the transmission of cell structure variants in isogenic lines of single-celled eukaryotes (Beisson and Sonneborn 1965; Nanney 1968), continued to accumulate, such effects were largely neglected by biologists and medical researchers until fairly recently (Sapp 1987; Jablonka and Lamb 1995; Bonduriansky and Day 2018). Nongenetic inheritance began to be rediscovered during the 1980s and 1990s through accumulating evidence of epigenetic inheritance in plants and rodents (Jablonka and Lamb 1995), reports of maternal and paternal environment effects in a variety of plant and animal systems (Bernardo 1996; Mousseau and Fox 1998), and increasing recognition of the importance of parental environment and lifestyle for children's health in humans (Pembrey et al. 2006; Gluckman et al. 2007, 2009; Pembrey et al. 2014).

While just about everyone now acknowledges that transgenerational plasticity is a real and widespread phenomenon, not everyone is convinced that transgenerational plasticity could play an important role in evolution. Some evolutionary geneticists 
believe that adaptive evolution can be adequately understood through the traditional perspective of natural selection acting on genetic variation, and that plasticityeither within or across generations - is merely an evolved mechanism that should be viewed as a product of evolution rather than an independent factor that can influence the course of evolution. Proponents of this traditional gene-centric view do not deny that transgenerational plasticity exists, but they tend to downplay its scope and importance. In particular, traditionalists argue that transgenerational plasticity cannot play the same role as genetic mutation in furnishing variation on which natural selection can act because environment-induced traits tend to be less stable than genetic alleles, and because the range of variation that can be induced by environment may be limited to the presence or absence of one particular phenotype. By contrast, some researchers believe that transgenerational plasticity could play a very important role in generating heritable variation and influencing the course of evolution. They point out that examples of highly stable induced phenotypes exist, and that novel environments could induce a broad range of novel developmental responses on which natural selection could act. Moreover, as I explain below, theory suggests that even instances of transgenerational plasticity that do not persist beyond the offspring generation can still have a very substantial influence on offspring development, and thereby influence natural selection on genetic alleles.

If transgenerational plasticity is viewed as a source of heritable variation, then non-adaptive transgenerational plasticity could be just as interesting and important as adaptive transgenerational plasticity. Genetic mutation is an essentially nonadaptive process, but it plays a central role in adaptive evolution by providing a pool of variation for natural selection to act on. It has been suggested that novel forms of within-generation plasticity, expressed in novel environments that reveal cryptic genetic variation and thereby induce a range of novel phenotypes, could play a similar role (reviewed in West-Eberhard 2003; see Futuyma 2021; Levis and Pfennig 2021; and Pfennig 2021 in this volume for a discussion of the history of these ideas). If genotypes express different reaction norms across an environmental gradient, and if some reaction norms bring the phenotype closer to the adaptive peak in the new environment, then selection can favor those genotypes. Moreover, if the new conditions are stable, genetic assimilation of the optimal phenotype can occur (see Scheiner and Levis 2021 in this volume). Similarly, if a novel environment could induce novel forms of transgenerational plasticity, those that enhance fitness could be stabilized via genetic assimilation (see Pigliucci et al. 2006). In this way, forms of transgenerational plasticity (or within-generation plasticity) that were non-adaptive or even maladaptive ancestrally could provide the basis for new, adaptive evolutionary responses. Discussion continues on the role of within-generation plasticity in adaptive evolution, and a number of hypotheses have been proposed (e.g., see Levis and Pfennig 2021; Pfennig 2021 in this volume). However, while many of these ideas also apply to transgenerational plasticity, the role of transgenerational plasticity in adaptive evolution is likely to differ in some ways from that of within-generation plasticity.

First, some forms of transgenerational plasticity can have phenotypic effects that accumulate or interact over multiple generations. If the same environment is 
encountered in successive generations, the magnitude of a transgenerational plasticity effect can sometimes increase over several generations, or the effect in one generation can be modulated by effects in the next (Herman et al. 2012; Wylde et al. 2019; Tariel et al. 2020). Selection can therefore act not only on the transgenerational reaction norm (i.e., the function relating a phenotypic trait in descendants to an environmental factor experienced by their ancestors), but also on the way that factors transmitted from parents, grandparents, and even earlier ancestors are integrated in development.

Second, transgenerational plasticity effects can interact with genetic alleles to bring about adaptive evolution. For example, in some insects, males that encounter abundant nutrients during the larval stage develop into large adults that express enlarged secondary sexual traits, and such males also sire larger offspring (Hunt and Simmons 2000; Bonduriansky and Head 2007). Such condition transfer from males to their offspring could select for genetically based female preferences for highcondition males: even if genetic variation in condition is lacking, females can benefit by mating with high-condition males because such males transmit their acquired condition to their offspring (Bonduriansky and Day 2013). Similar effects can occur in the context of host-parasite coevolution. If hosts acquire and transmit to their offspring some degree of resistance to infection, then this form of transgenerational plasticity will select for genetic or nongenetic parasite traits that can overcome the acquired resistance (Gomez-Diaz et al. 2012; Mukherjee et al. 2019). Likewise, if deleterious effects of an environmental stressor are more likely to be transmitted by older parents, resulting in environment-dependent parental age effects, then a change in the environment that results in increased stress might result in declining phenotypic quality and select for alleles that bring about earlier reproduction (Bonduriansky and Day 2018). In this way, a deleterious form of transgenerational plasticity can drive adaptive evolution of life history.

Importantly, such interactions between environment-induced nongenetic factors and genetic alleles can occur even when transgenerational plasticity is limited to single-generation (parent to offspring) transmission. Indeed, because transient environmental effects that are induced de novo by environment in every generation represent heritable variation that cannot be depleted by selection, such transient nongenetic factors have the greatest potential to influence genetic evolution (Bonduriansky and Day 2013). Theory therefore suggests that both stable and transient environment-induced effects can play important but distinct roles in adaptive evolution. Relatively stable nongenetic factors that can persist over multiple generations (such as the peloric epiallele of the Lcyc gene that influences flower form) could provide abundant heritable variation on which selection can act, potentially allowing for rapid adaptation to a novel environment (Day and Bonduriansky 2011; Bonduriansky et al. 2012; Klironomos et al. 2013; Furrow 2014). Transient nongenetic factors that can only be transmitted over a single generation (such as effects of acquired condition on offspring growth) could interact with more stable nongenetic factors or with genetic alleles to influence the course of evolution (Feldman and Cavalli-Sforza 1989; Richerson and Boyd 2005; Bonduriansky and Day 2013).

Understanding the evolutionary role of transgenerational plasticity will therefore require a combination of empirical and theoretical research. We need data on the potential for transgenerational plasticity effects to accumulate and interact over 
multiple generations, the potential for novel environments to induce the expression of novel plastic phenotypes, and the potential for formerly deleterious transgenerational plasticity effects to enhance fitness in novel environments. At the same time, theoretical research is required to identify potential contexts in which transgenerational plasticity can interact with genetic variation to drive adaptive evolution. The available evidence is frustratingly sparse because the studies required to answer these questions have rarely been attempted (see Futuyma 2021 in this volume). Much more work is needed to test ideas and clarify the role of transgenerational plasticity in adaptive evolution. In Box 13.2, I outline some questions for future research on transgenerational plasticity.

\section{BOX 13.2 SUGGESTIONS FOR FUTURE RESEARCH}

- Under what conditions does transgenerational plasticity evolve?

- Over how many generations can environment-induced effects persist?

- What proximate mechanisms mediate adaptive transgenerational plasticity, and what are the metabolic/physiological costs of those mechanisms?

- What proximate mechanisms mediate non-adaptive transgenerational plasticity?

- What are the conditions that select for anticipatory versus condition-transfer forms of adaptive transgenerational plasticity?

- Under what circumstances does selection favor genetic assimilation of phenotypes ancestrally engendered by adaptive or non-adaptive transgenerational plasticity, and how much does this process contribute to adaptive evolution?

- Under what circumstances is transgenerational plasticity subject to parent-offspring or sexual conflicts?

\section{REFERENCES}

Agrawal, A. A., C. Laforsch, and R. Tollrian. 1999. Transgenerational induction of defences in animals and plants. Nature 401:60-63.

Amarger, V., A. Lecouillard, L. Ancellet, I. Grit, B. Castellano, P. Hulin, and P. Parnet. 2014. Protein content and methyl donors in maternal diet interact to influence the proliferation rate and cell fate of neural stem cells in rat hippocampus. Nutrients 6:4200-4217.

Anderson, L. M., L. Riffle, R. Wilson, G. S. Travlos, M. S. Lubomirski, and W. G. Alvord. 2006. Preconceptional fasting of fathers alters serum glucose in offspring of mice. Nutrition 22:327-331.

Aplin, L. M. 2019. Culture and cultural evolution in birds: A review of the evidence. Animal Behaviour 147:179-187.

Archer, E. 2015. The childhood obesity epidemic as a result of nongenetic evolution: The maternal resources. Mayo Clinic Proceedings 90:77-92.

Beisson, J. and T. M. Sonneborn. 1965. Cytoplasmic inheritance of the organization of the cell cortex in Paramecium Aurelia. Proceedings of the National Academy of Sciences of the United States of America 53:275-282. 
Bell, A. M. and J. K. Hellmann. 2019. An integrative framework for understanding the mechanisms and multigenerational consequences of transgenerational plasticity. Annual Review of Ecology, Evolution, and Systematics 50:97-118.

Bernardo, J. 1996. Maternal effects in animal ecology. American Zoologist 36:83-105.

Bonduriansky, R. 2012. Rethinking heredity, again. Trends in Ecology \& Evolution 27:330-336.

Bonduriansky, R. and A. J. Crean. 2018. What are parental condition-transfer effects and how can they be detected? Methods in Ecology and Evolution 9:450-456.

Bonduriansky, R. and T. Day. 2013. Nongenetic inheritance and the evolution of costly female preference. Journal of Evolutionary Biology 26:76-87.

Bonduriansky, R. and T. Day. 2018. Extended Heredity: A New Understanding of Inheritance and evolution. Princeton University Press, Princeton, NJ.

Bonduriansky, R. and M. Head. 2007. Maternal and paternal condition effects on offspring phenotype in Telostylinus angusticollis (Diptera: Neriidae). Journal of Evolutionary Biology 20:2379-2388.

Bonduriansky, R., A. J. Crean, and T. Day. 2012. The implications of nongenetic inheritance for evolution in changing environments. Evolutionary Applications 5:192-201.

Boskovic, A. and O. J. Rando. 2018. Transgenerational epigenetic inheritance. Annual Review of Genetics 52:21-41.

Burke, N. W., S. Nakagawa, and R. Bonduriansky. 2020. Sexual conflict explains diverse patterns of transgenerational plasticity. bioRxiv.

Champagne, F. A. and M. J. Meaney. 2007. Transgenerational effects of social environment on variations in maternal care and behavioral response to novelty. Behavioral Neuroscience 121:1353-1363.

Chan, J. C., C. P. Morgan, N. Adrian Leu, A. Shetty, Y. M. Cisse, B. M. Nugent, K. E. Morrison, E. Jašarević, W. Huang, N. Kanyuch, A. B. Rodgers, N. V. Bhanu, D. S. Berger, B. A. Garcia, S. Ament, M. Kane, C. Neill Epperson, and T. L. Bale. 2020. Reproductive tract extracellular vesicles are sufficient to transmit intergenerational stress and program neurodevelopment. Nature Communications 11:1499.

Chen, T. H., Y. H. Chiu, and B. J. Boucher. 2006. Transgenerational effects of betel-quid chewing on the development of the metabolic syndrome in the Keelung Communitybased Integrated Screening Program. The American Journal of Clinical Nutrition 83:688-692.

Colicchio, J. 2017. Transgenerational effects alter plant defense and resistance in nature. Journal of Evolutionary Biology 30:664-680.

Crean, A. J. and R. Bonduriansky. 2014. What is a paternal effect? Trends in Ecology \& Evolution 29:554-559.

Crean, A. J., J. M. Dwyer, and D. J. Marshall. 2013. Adaptive paternal effects? Experimental evidence that the paternal environment affects offspring performance. Ecology 94:2575-2582.

Cubas, P., C. Vincent, and E. Coen. 1999. An epigenetic mutation responsible for natural variation in floral symmetry. Nature 401:157-161.

Darwin, C. R. 1859. On the Origin of Species. John Murray, London, UK.

Darwin, C. R. 1871. The Descent of Man. Murray, London, UK.

Darwin, C. R. 1875. The Variation of Animals and Plants under Domestication. John Murray, London, UK.

Day, T. and R. Bonduriansky. 2011. A unified approach to the evolutionary consequences of genetic and nongenetic inheritance. The American Naturalist 178:E18-E136.

Dey, S., S. R. Proulx, and H. Teotonio. 2016. Adaptation to temporally fluctuating environments by the evolution of maternal effects. PLoS Biology 14:e1002388.

Diamond, S. E. and R. A. Martin. 2021. Buying time: Plasticity and population persistence. In D. W. Pfennig, ed., Phenotypic Plasticity and Evolution: Causes, Consequences, Controversies. CRC Press, Boca Raton, FL. 
Dominguez-Salas, P., S. E. Moore, M. S. Baker, A. W. Bergen, S. E. Cox, R. A. Dyer, A. J. Fulford, Y. Guan, E. Laritsky, M. J. Silver, G. E. Swan, S. H. Ziesel, S. M. Innis, R. A. Waterland, A. M. Prentice, and B. J. Hennig. 2014. Maternal nutrition at conception modulates DNA methylation of human metastable epialleles. Nature Communications 5:3746.

Donelson, J. M., P. L. Muday, and M. I. McCormick. 2009. Parental effects on offspring life histories: When are they important? Biology Letters 23:262-265.

Donkin, I., S. Versteyhe, L. R. Ingerslev, K. Qian, M. Mechta, L. Nordkap, B. Mortensen, E. V. Appel, N. Jorgensen, V. B. Kristiansen, T. Hansen, C. T. Workman, J. R. Zierath, and R. Barres. 2016. Obesity and bariatric surgery drive epigenetic variation of spermatozoa in humans. Cell Metabolism 23:369-378.

Duncan, E. J., P. D. Gluckman, and P. K. Dearden. 2014. Epigenetics, plasticity and evolution: How do we link epigenetic change to phenotype? Journal of Experimental Zoology Part B Molecular and Developmental Evolution 322B:208-220.

Engqvist, L. and K. Reinhold. 2016. Adaptive transgenerational phenotypic plasticity and the lack of an experimental control in reciprocal match/mismatch experiments. Methods in Ecology and Evolution 7:1482-1488.

Feldman, M. W. and L. L. Cavalli-Sforza. 1989. On the theory of evolution under genetic and cultural transmission with application to the lactose absorption problem, pp. 145-173. In M. W. Feldman, ed., Mathematical Evolutionary Theory. Princeton University Press, Princeton, NJ.

Fox, C. W., M. S. Thakar, and T. A. Mousseau. 1997. Egg size plasticity in a seed beetle: An adaptive maternal effect. The American Naturalist 149:149-163.

Furrow, R. E. 2014. Epigenetic inheritance, epimutation, and the response to selection. PLoS One 9:e101559.

Futuyma, D. J. 2021. How does phenotypic plasticity fit into evolutionary theory? In D. W. Pfennig, ed., Phenotypic Plasticity and Evolution: Causes, Consequences, Controversies. CRC Press, Boca Raton, FL.

Galloway, L. F. and J. R. Etterson. 2007. Transgenerational plasticity is adaptive in the wild. Science 318:1134-1136.

Gijzen, M., C. Ishmael, and S. D. Shrestha. 2014. Epigenetic control of effectors in plant pathogens. Frontiers in Plant Science 5:638.

Gluckman, P. D., M. A. Hanson, and A. S. Beedle. 2007. Non-genomic transgenerational inheritance of disease risk. Bioessays 29:145-154.

Gluckman, P. D., M. A. Hanson, T. Buklijas, F. M. Low, and A. S. Beedle. 2009. Epigenetic mechanisms that underpin metabolic and cardiovascular diseases. Nature Reviews Endocrinology 5:401-408.

Gomez-Diaz, E., M. Jorda, M. A. Peinado, and A. Rivero. 2012. Epigenetics of host-pathogen interactions: The road ahead and the road behind. PLoS Pathogens 8:e100300.

Greer, E. L., T. J. Maures, A. G. Hauswirth, E. M. Green, D. S. Leeman, G. S. Maro, S. Han, M. R. Banko, O. Gozani, and A. Brunet. 2010. Members of theH3K4 trimethylation complex regulate lifespan in a germline-dependent manner in C. elegans. Nature 466:383-387.

Greer, E. L., T. J. Maures, D. Ucar, A. G. Hauswirth, E. Mancini, J. P. Lim, B. A. Benayoun, Y. Shi, and A. Brunet. 2011. Transgenerational epigenetic inheritance of longevity in Caenorhabditis elegans. Nature 479:365-371.

Gribble, K. E., G. Jarvis, M. Bock, and D. B. M. Welch. 2014. Maternal caloric restriction partially rescues the deleterious effects of advanced maternal age on offspring. Aging Cell 13:623-630.

Hellmann, J. K., E. R. Carlson, and A. M. Bell. 2020. Grandpaternal effects are lineage- and sex-specific in threespined sticklebacks. bioRxiv.

Herman, J. J., S. E. Sultan, T. Horga-Kobelski, and C. Riggs. 2012. Adaptive transgenerational plasticity in an annual plant: Grandparental and parental drought stress enhance performance of seedlings in dry soil. Integrative \& Comparative Biology 52:77-88. 
Hunt, J. and L. W. Simmons. 2000. Maternal and paternal effectson offspring phenotype in the dung beetle Onthophagus taurus. Evolution 54:936-941.

Jablonka, E. and M. J. Lamb. 1995. Epigenetic Inheritance and Evolution. Oxford University Press, Oxford, UK.

Jablonka, E. and G. Raz. 2009. Transgenerational epigenetic inheritance: Prevalence, mechanisms, and implications for the study of heredity and evolution. Quarterly Review of Biology 84:131-176.

Jesmer, B. R., J. A. Merkle, J. R. Goheen, E. O. Aikens, J. L. Beck, A. B. Courtemanch, M. A. Hurley, D. E. McWhirter, H. M. Miyasaki, K. L. Monteith, and M. J. Kauffman. 2018. Is ungulate migration culturally transmitted? Evidence of social learning from translocated animals. Science 361:1023-1025.

Kamimae-Lanning, A. N., S. M. Krasnow, N. A. Goloviznina, X. Zhu, Q. R. Roth-Carter, P. R. Levasseur, S. Jeng, S. K. McWeeney, P. Kurre, and D. L. Marks. 2014. Maternal highfat diet and obesity compromise fetal hematopoiesis. Molecular Metabolism 4:25e38.

Kirkpatrick, M. and R. Lande. 1989. The evolution of maternal characters. Evolution 43:485-503.

Klironomos, F. D., J. Berg, and S. Collins. 2013. How epigenetic mutations can affect genetic evolution: Model and mechanism. Bioessays 35:571-578.

Klosin, A., E. Casas, C. Hidalgo-Carcedo, T. Vavouri, and B. Lehner. 2017. Transgenerational transmission of environmental information in C. elegans. Science 356:320-323.

Kuijper, B. and R. A. Johnstone. 2018. Maternal effects and parent-offspring conflict. Evolution 72:220-233.

Lachmann, M. and E. Jablonka. 1996. The inheritance of phenotypes: An adaptation to fluctuating environments. Journal of Theoretical Biology 181:1-9.

Laforsch, C. and R. Tollrian. 2004. Inducible defenses in multipredator environments: cyclomorphosis in Daphnia cucullata. Ecology 85:2302-2311.

Lamarck, J.-B. 1809. Philosophie Zoologique. MacMillan and Co., London, UK.

Leimar, O. and J. M. McNamara. 2015. The evolution of transgenerational integration of information in heterogeneous environments. The American Naturalist 185:E55-E69.

Levis, N. A. and D. W. Pfennig. 2021. Innovation and diversification via plasticity-led evolution. In D. W. Pfennig, ed., Phenotypic plasticity and evolution: Causes, consequences, controversies. CRC Press, Boca Raton, FL.

Macartney, E. L., A. J. Crean, and R. Bonduriansky. 2018. Epigenetic paternal effects as costly, condition-dependent traits. Heredity 121:248-256.

Marshall, D. J. and T. Uller. 2007. When is a maternal effect adaptive? Oikos 116:1957-1963.

Mashoodh, R. and F. A. Champagne. 2014. Paternal epigenetic inheritance, pp. 221-235. In T.O. Tollefsbol, ed., Transgenerational epigenetics, Academic Press, Cambridge, MA.

McGhee, K. E., L. M. Pintor, E. L. Suhr, and A. M. Bell. 2012. Maternal exposure to predation risk decreases offspring antipredator behaviour and survival in threespined stickleback. Functional Ecology 26:932-940.

McNamara, J. M., S. R. Dall, P. Hammerstein, and O. Leimar. 2016. Detection versus selection: Integration of genetic, epigenetic and environmental cues in fluctuating environments. Ecology Letters 19:1267-1276.

Mesoudi, A. 2011. Cultural Evolution: How Darwinian Theory can Explain Human Culture and Synthesize the Social Sciences. The University of Chicago Press, Chicago, IL.

Monaghan, P. and N. B. Metcalfe. 2019. The deteriorating soma and the indispensable germline: Gamete senescence and offspring fitness. Proceedings of the Royal Society B 286:20192187.

Mousseau, T. A. and C. W. Fox. 1998. The adaptive significance of maternal effects. Trends in Ecology and Evolution 13:403-407.

Mukherjee, K., I. Dubovskiy, E. Grizanova, R. Lehmann, and A. Vilcinskas. 2019. Epigenetic mechanisms mediate the experimental evolution of resistance against parasitic fungi in the greater wax moth Galleria mellonella. Scientific Reports 9:1626. 
Murren, C. J., J. R. Auld, H. Callahan, C. K. Ghalambor, C. A. Handelsman, M. A. Heskel, J. G. Kingsolver, H. J. Maclean, J. Masel, H. Maughan, D. W. Pfennig, R. A. Relyea, S. Seiter, E. Snell-Rood, U. K. Steiner, and C. D. Schlichting. 2015. Constraints on the evolution of phenotypic plasticity: Limits and costs of phenotype and plasticity. Heredity 115:293-301.

Nanney, D. L. 1968. Cortical patterns in cellular morphogenesis. Science 160:496-502.

Ng, S.-F., R. C. Y. Lin, D. R. Laybutt, R. Barres, J. A. Owens, and M. J. Morris. 2010. Chronic high-fat diet in fathers programs $\beta$-cell dysfunction in female rat offspring. Nature 467:963-967.

O’Brien, E. A., K. S. Ensbey, B. W. Day, P. A. Baldock, and G. Barry. 2020. Direct evidence for transport of RNA from the mouse brain to the germline and offspring. BMC Biology $18: 45$.

O’Dea, R. E., R. Vega-Trejo, M. L. Head, and M. D. Jennions. 2015. Maternal effects on offspring size and number in mosquitofish, Gambusia holbrooki. Ecology and Evolution 5:2945-2955.

Paksa, A. and J. Rajagopal. 2017. The epigenetic basis of cellular plasticity. Current Opinion in Cell Biology 49:116-122.

Pál, C. and I. Miklós. 1999. Epigenetic inheritance, genetic assimilation and speciation. Journal of Theoretical Biology 200:19-37.

Pembrey, M. E., L. O. Bygren, G. Kaati, S. Edvinsson, K. Northstone, M. Sjostrom, J. Golding, and A. S. Team. 2006. Sex-specific, male-line transgenerational responses in humans. European Journal of Human Genetics 14:159-166.

Pembrey, M., R. Saffery, L. O. Bygren, and Network in Epigenetic Epidemiology. 2014. Human transgenerational responses to early-life experience: Potential impact on development, health and biomedical research. Journal of Medical Genetics 51:563-572.

Pfennig, D. W. 2021. Key questions about phenotypic plasticity. In D. W. Pfennig, ed., Phenotypic Plasticity and Evolution: Causes, Consequences, Controversies. CRC Press, Boca Raton, FL.

Pfennig, D. W. and M. R. Servedio. 2012. The role of transgenerational epigenetic inheritance in diversification and speciation. Non-Genetic Inheritance 2012:17-26.

Piersma, T. and J. Drent. 2003. Phenotypic flexibility and the evolution of organismal design. Trends in Ecology and Evolution 18:228-233.

Pigliucci, M., C. J. Murren, and C. D. Schlichting. 2006. Phenotypic plasticity and evolution by genetic assimilation. Journal of Experimental Biology 209:2362-2367.

Proulx, S. R. and H. Teotonio. 2017. What kind of maternal effects can be selected for in fluctuating environments? The American Naturalist 189:E118-E137.

Qutob, D., B. P. Chapman, and M. Gijzen. 2013. Transgenerational gene silencing causes gain of virulence in a plant pathogen. Nature Communications 4:1349.

Qvarnström, A. and T. D. Price. 2001. Maternal effects, paternal effects and sexual selection. Trends in Ecology \& Evolution 16 95-100.

Rechavi, O. and I. Lev. 2017. Principles of transgenerational small RNA inheritance in caenorhabditis elegans. Current Biology 27:R720-R730.

Richerson, P. J. and R. Boyd. 2005. Not by Genes Alone: How Culture Transforms Human Evolution. The University of Chicago Press, Chicago, IL.

Rutkowska, J., M. Lagisz, R. Bonduriansky, and S. Nakagawa. 2020. Mapping the past, present and future research landscape of paternal effects. BMC Biology 18:183.

Sapp, J. 1987. Beyond the Gene: Cytoplasmic Inheritance and the Struggle for Authority in Genetics. Oxford University Press, Oxford, UK.

Scheiner, S. M. and N. A. Levis. 2021. The loss of phenotypic plasticity via natural selection: Genetic assimilation. In D. W. Pfennig, ed., Phenotypic Plasticity and Evolution: Causes, Consequences, Controversies. CRC Press, Boca Raton, FL.

Schmauss, C., Z. Lee-McDermott, and L. R. Medina. 2014. Trans-generational effects of early life stress: The role of maternal behaviour. Scientific Reports 4:4873. 
Sharp, G. C. and D. A. Lawlor. 2019. Paternal impact on the life course development of obesity and type 2 diabetes in the offspring. Diabetologia 62:1802-1810.

Skinner, M. K. 2014. Environmental stress and epigenetic transgenerational inheritance. BMC Medicine 12:153.

Snell-Rood, E. and S. Ehlman. 2021. Ecology and evolution of plasticity. In D. W. Pfennig, ed. Phenotypic Plasticity and Evolution: Causes, Consequences, Controversies. CRC Press, Boca Raton, FL.

Soubry, A., C. Hoyo, R. L. Jirtle, and S. K. Murphy. 2014. A paternal environmental legacy: Evidence for epigenetic inheritance through the male germ line. Bioessays 36:359-371.

Tariel, J., S. Plenet, and E. Luquet. 2020. Transgenerational plasticity of inducible defences: Combined effects of grand-parental, parental and current environments. Ecology and Evolution 10(5):2367-2376.

Thompson, M. E., M. N. Muller, K. Sabbi, Z. P. Machanda, E. Otali, and R. W. Wrangham. 2016. Faster reproductive rates trade off against offspring growth in wild chimpanzees. Proceedings of the National Academy of Sciences USA 113:7780-7785.

Uller, T. 2008. Developmental plasticity and the evolution of parental effects. Trends in Ecology \& Evolution 23:432-438.

Van Buskirk, J. and U. K. Steiner. 2009. The fitness costs of developmental canalization and plasticity. Journal of Evolutionary Biology 22:852-860.

Van Leeuwen, T. E., D. McLennan, S. McKelvey, D. C. Stewart, C. E. Adams, and N. B. Metcalfe. 2016. The association between parental life history and offspring phenotype in Atlantic salmon. Journal of Experimental Biology 219:374-382.

War, A. R., M. G. Paulraj, T. Ahmad, A. A. Buhroo, B. Hussain, S. Ignacimuthu, and H. C. Sharma. 2012. Mechanisms of plant defense against insect herbivores. Plant Signaling \& Behavior 7:1306-1132.

Weaver, I. C. G., N. Cervoni, F. A. Champagne, A. C. D’Alessio, S. Sharma, J. R. Seckl, S. Dymov, M. Szyf, and M. J. Meaney. 2004. Epigenetic programming by maternal behavior. Nature Neuroscience 7:847-854.

West-Eberhard, M. J. 2003. Developmental Plasticity and Evolution. Oxford University Press, Oxford, UK.

Wund, M. A., J. A. Baker, J. L. Golub, and S. A. Foster. 2015. The evolution of antipredator behaviour following relaxed and reversed selection in Alaskan threespine stickleback fish. Animal Behaviour 106:181-189.

Wylde, Z., F. Spagopoulou, A. K. Hooper, A. A. Maklakov, and R. Bonduriansky. 2019. Parental breeding age effects on descendants' longevity interact over two generations in matrilines and patrilines. PLoS Biology 17:e3000556.

Youngson, N. A. and E. Whitelaw. 2008. Transgenerational epigenetic effects. Annual Review of Genomics and Human Genetics 9:233-257.

Zannas, A. S., J. Arloth, T. Carrillo-Roa, S. Iurato, S. Röh, K. J. Ressler, C. B. Nemeroff, A. K. Smith, B. Bradley, C. Heim, A. Menke, J. F. Lange, T. Brückl, M. Ising, N. R. Wray, A. Erhardt, E. B. Binder, and D. Mehta. 2015. Lifetime stress accelerates epigenetic aging in an urban, African American cohort: Relevance of glucocorticoid signaling. Genome Biology 16:266. 